\title{
Anaerobic brain abscess following chronic suppurative otitis media in a child from Uganda
}

\author{
KA Cox MD, G Al-Rawahi MD, TR Kollmann MD PhD
}

\begin{abstract}
KA Cox, G Al-Rawahi, TR Kollmann. Anaerobic brain abscess following chronic suppurative otitis media in a child from Uganda. Can J Infect Dis Med Microbiol 2008;19(3):e91-e93.

Brain abscess, while rare, confers high mortality, especially in the developing world. The case of a Ugandan child with a polymicrobial brain abscess including infection with Tissierella praeacuta/Clostridium hastiforme requiring repeated drainage and eventual surgical excision is reported. The case demonstrates the importance of considering anaerobic organisms in the treatment of children with brain abscess from the developing world.
\end{abstract}

Key Words: Brain abscess; Clostridium hastiforme; Otitis media; Tissierella praeacuta

\section{CASE PRESENTATION}

A 10-year-old boy from Uganda traveling through Vancouver, British Columbia, on a choir tour presented with 10 days of vomiting and abdominal pain. Over the preceding two days, he had complained of headache and developed poor balance, polyuria and polydipsia. On the day of presentation, he awoke with left-sided weakness and left-sided facial droop. He had a four-month history of recurrent drainage from his left ear. While in Uganda, he had been treated with multiple courses of antibiotics. He had a history of recurrent cough, poor weight gain and hearing loss. Otherwise, his medical history was unremarkable. His parents had died of an unknown illness and he had been cared for in a Ugandan orphanage for the past three years. His immunizations (World Health Organization Expanded Program on Immunisation schedule) were up to date. He was in significant pain, pointing to his head. He displayed signs of meningismus as well as drooping of his right eyelid and mild left upper and lower facial droop. Pupillary reflexes were normal with slight reduction in left eye abduction. Tone was reduced in both the left upper and lower limbs with decreased strength in the left upper and lower limbs. Reflexes were normal. His right tympanic membrane was erythematous and bulging with purulent discharge. A culture from the ear canal isolated Morganella morganii, Proteus mirabilis, Corynebacterium amycolatum and Enterobacter cloacae; however, an anaerobic culture was not set up. The white blood cell count was $9.7 \times 10^{9} / \mathrm{L}$ (neutrophils $53 \%$, lymphocytes $31 \%$, monocytes $15 \%$, eosinophils 1\%). Malaria smears, HIV serology, and tuberculosis smear and culture were negative. A computed tomography scan showed a nonenhancing large fluid-filled mass in the right parietal lobe with midline shift (Figure 1). He was taken to the operating room for drainage of the abscess and started on intravenous meropenem (120 mg/kg/day, every $8 \mathrm{~h})$. A Gram-stained smear of the abscess fluid showed two

\section{Un abcès cérébral anaérobique après une otite moyenne suppurative chez un enfant de l'Ouganda}

\begin{abstract}
L'abcès cérébral, même s'il est rare, confère un fort taux de mortalité, notamment dans les pays en voie de développement. On expose le cas d'un enfant ougandais ayant un abcès cérébral polymicrobien, y compris une infection par le Tissierella praeacuta et le Clostridium hastiforme exigeant un drainage répété, puis finalement une excision chirurgicale. Le cas démontre l'importance d'envisager la présence d'organismes anaérobiques dans le traitement des enfants ayant un abcès cérébral et provenant de pays en voie de développement.
\end{abstract}

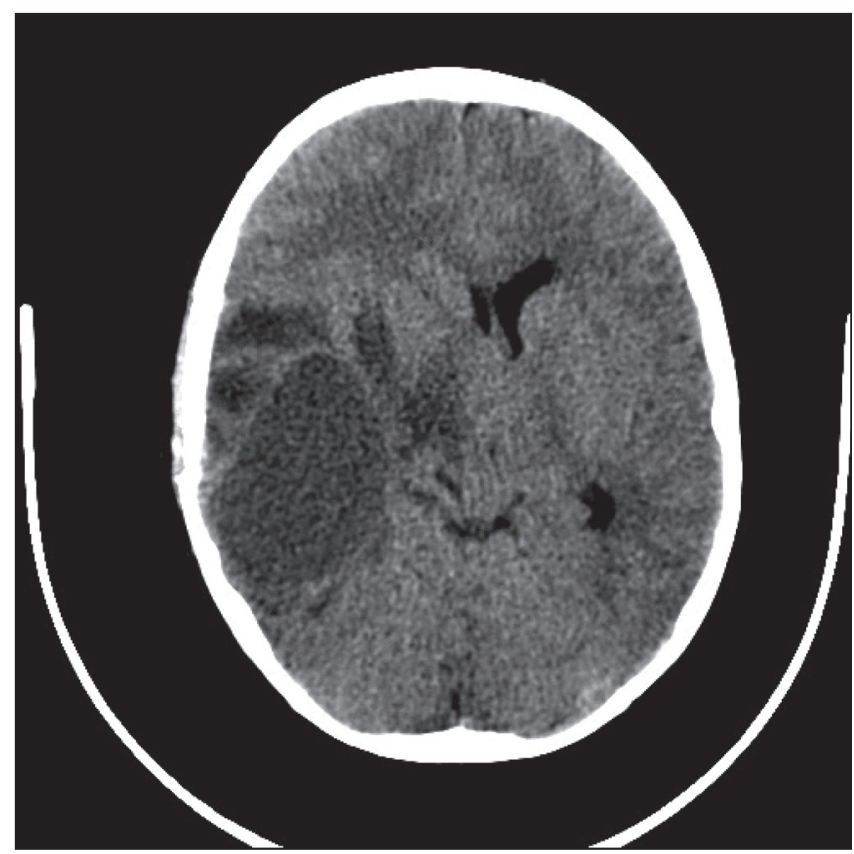

Figure 1) Computed tomography scan of patient with a nonenhancing fluid-filled lesion in the right parietal lobe with midline shift of brain

morphotypes of Gram-negative bacilli and large Gram-positive bacilli with terminal spores. The culture demonstrated growth of Proteus mirabilis, Bacteroides fragilis group, and anaerobic large Gram-positive bacilli with terminal spores. The later isolate was sent to the reference lab (BC Centre for Disease Control) for identification and was identified as Clostridium hastiforme using biochemical tests. After three failed attempts at percutaneous drainage, the abscess was excised by craniotomy. Following this, the patient slowly recovered full function. 


\section{DISCUSSION}

Although brain abscesses are rare, they confer significant morbidity and mortality. Brain abscess constitutes $1 \%$ to $2 \%$ of all space-occupying lesions in the developed world and up to $8 \%$ in the developing world (1). Over the past 40 years, improved surgical techniques, effective antimicrobials and modern imaging techniques have reduced mortality from $30 \%$ to $60 \%$ to $10 \%(1,2)$; however, in some countries, the mortality is still as high as $30 \%$ (3).

A review of 107 cases of brain abscess in children younger than 15 years of age found congenital heart disease and chronic otitis media to be the most common predisposing factors, accounting for $72 \%$ of cases (4). Organisms identified as contributing to otitis media-related brain abscesses were Proteus species in $38.1 \%$ and Pseudomonas species in 19.1\% (4). In these series, there were no cases of Clostridium-related abscesses; however, only 96 had a bacteriological investigation performed and there is no indication if anaerobic cultures were obtained from all of these specimens.

Although identified as $\mathrm{C}$ hastiforme by the reference lab, the current taxonomy of this organism is Tissierella praeacuta (5). In this report we will refer to it as $\mathrm{T}$ praeacuta/C hastiforme.

A report of forty-five cases of clostridial brain abscesses isolated C perfringens in 32 cases (71\%) and C septicum in seven cases $(16 \%)(6)$. The majority were related to penetrating trauma $(n=25 ; 56 \%)$. The rest were associated with cancer $(13 \%)$, postcraniotomy $(11 \%)$, otitis media $(9 \%)$ and one case was related to hemolytic uremic syndrome (6). Other Clostridium species causing brain abscesses include $\mathrm{C}$ tertium (7), C bifermentans (8), C septicum (98), C beijerinckii and C butyricum (10). There are four cases of Clostridium species isolated from brain abscess secondary to otomastoiditis, out of which three involved C perfringens (10-13). In one case, of an 11-year-old with acute otomastoiditis, the abscess culture grew $\mathrm{C}$ butyricum or beijerinckii along with Streptococcal species (10).

To our knowledge, this is first reported case of $T$ praeacuta/C hastiforme causing a central nervous system abscess in humans. However, it is likely that cases have been missed, due to the stringent anaerobic culture requirements. $T$ praeacuta/C hastiforme has been reported in two previous case reports, one of gas gangrene of the eyelid (14) and the other of colonization in a four-year-old boy with combined variable immune deficiency (15).

\section{REFERENCES}

1. Prasad KN, Mishra AM, Gupta D, Husain N, Husain M, Gupta RK. Analysis of microbial etiology and mortality in patients with brain abscess. J Infect 2006;53:221-7.

2. Rosenblum ML, Hoff JT, Norman D, Weinstein PR, Pitts L. Decreased mortality from brain abscesses since advent of computerized tomography. J Neurosurg 1978;49:658-68.

3. Qureshi HU, Habib AA, Siddiqui AA, Mozaffar T, Sarwari AR. Predictors of mortality in brain abscess. J Pak Med Assoc 2002;52:111-6.

4. Auvichayapat N, Auvichayapat P, Aungwarawong S. Brain abscess in infants and children: A retrospective study of 107 patients in northeast Thailand. J Med Assoc Thai 2007;90:1601-7.

5. Bae JW, Park JR, Chang YH, Rhee SK, Lim BC, Park YH. Clostridium hastiforme is a later synonym of Tissierella praeacuta. Int J Syst Evol Microbiol 2004;54:947-9.

6. Colen CB, Rayes M, Rengachary S, Guthikonda M. Outcome of brain abscess by Clostridium perfringens. Neurosurgery. 2007;61:E1339.
The source of the $T$ praeacuta/C hastiforme isolated from brain abscess in our patient remains unclear. The caregiver was not aware of any practices that would have resulted in contact with material potentially containing $\mathrm{T}$ praeacuta/C hastiforme. The Kikuyu of central Kenya have been known to install plant extracts mixed with chicken fat as a lubricant into ears affected with otitis media (16). It is unclear whether practices such as these might have exposed this patient to this unusual pathogen. In addition, in Malawi, a large proportion (91\%) of cultures from chronic suppurative otitis media (CSOM) grew fecal bacteria (17). The most common bacteria isolated in this series were Proteus mirabilis (74\% of ears) that was isolated in our patient as well, and Enteroccoccus species (60\%). In this series of cases from Malawi, dead flies were removed from many ear canals, and flies were hypothesized to be the main route of inoculation with fecal organisms. Given the polymicrobial spectrum grown from the abscess and the draining fluid in our case, a fecal source of the $T$ praeacuta/C hastiforme is indeed very likely.

It was previously thought that the common pathogens causing chronic serous otitis media include Staphylococcus aureus and Gram-negative enteric organisms, most commonly Pseudomonas aeruginosa. But in a recent review of the microbiology of CSOM in North America and Indonesia, anaerobes were identified in over $50 \%$ of cultures (18). The most commonly identified anaerobes were Peptostreptococcus species, anaerobic Gram-negative bacilli and Fusobacterium nucleatum. The previously infrequent identification of anaerobes in CSOM may be due to the challenging conditions required for collection and growth of these organisms (18). Anaerobic organisms must be considered as potential pathogens with CSOM, and metronidazole should be included in an empirical antibiotic regimen of such CSOM, especially if routine treatment is not effective.

ACKNOWLEDGEMENTS: TRK is supported in part by a Career Award in the Biomedical Sciences from the Burroughs Wellcome Fund, and by a CIHR Training Grant in Canadian Child Health Clinician Scientist Program, in partnership with SickKids Foundation, Child \& Family Research Institute (British Columbia), Women \& Children's Health Research Institute (Alberta), Manitoba Institute of Child Health.

7. Lew JF, Wiedermann BL, Sneed J, Campos J, McCullough D. Aerotolerant Clostridium tertium brain abscess following a lawn dart injury. J Clin Microbiol 1990;28:2127-9.

8. Pencek TL, Burchiel KJ. Delayed brain abscess related to a retained foreign body with culture of Clostridium bifermentans. J Neurosurg 1986;64:813-5.

9. Marangou AG, Joske RA, Kaard AO, Thomas W. Cerebral abscess due to Clostridium septicum. J R Soc Med 1992;85:641.

10. Jimenez-Mejias ME, Del Nozal M, Becceril B, Pichardo C, Polaina M, Arroyo A. Brain abscess caused by Clostridium sp. secondary to acute otomastoiditis. J Infect 2000;40:294-5.

11. Domingo Z. Clostridial brain abscesses. Br J Neurosurg.1994;8:691-4.

12. Morello A, Bettinazzi N. Brain abscess due to gas bacillus infection. Report of a case. J Neurosurg 1966;24:752-4.

13. Ubeda Sansano MI, Estan Capell M, Escribano Montaner A, Martinez Costa C, Hernandez Marco R. Cerebral abscess in childhood: A report of 5 cases. An Esp Pediatr 1996;45:177-80.

14. Lyon DB, Lemke BN. Eyelid gas gangrene. Ophthal Plast Reconstr Surg 1989;5:212-5. 
15. Williamson AP, Montgomery JR, South MA, Wilson R. A special report: Gour-year study of a boy with combined variable immunodeficiency maintained in strict reverse isolation from birth Pediatr Res 1977;11:63-9.

16. Njoroge GN, Bussmann RW. Traditional management of ear, nose and throat (ENT) diseases in Central Kenya. J Ethnobiol Ethnomed 2006;2:54.
17. van Hasselt $\mathrm{P}$, van Kregten E. Treatment of chronic suppurative otitis media with ofloxacin in hydroxypropyl methylcellulose ear drops: A clinical/bacteriological study in a rural area of Malawi. Int J Pediatr Otorhinolaryngol 2002;63:49-56.

18. Brook I. The role of anaerobic bacteria in chronic suppurative otitis media in children: Implications for medical therapy. Anaerobe 2008; 14:297-300. 


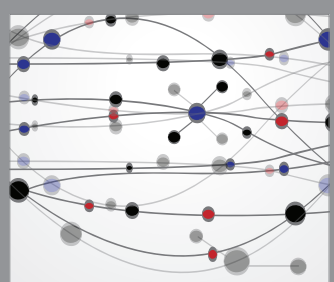

The Scientific World Journal
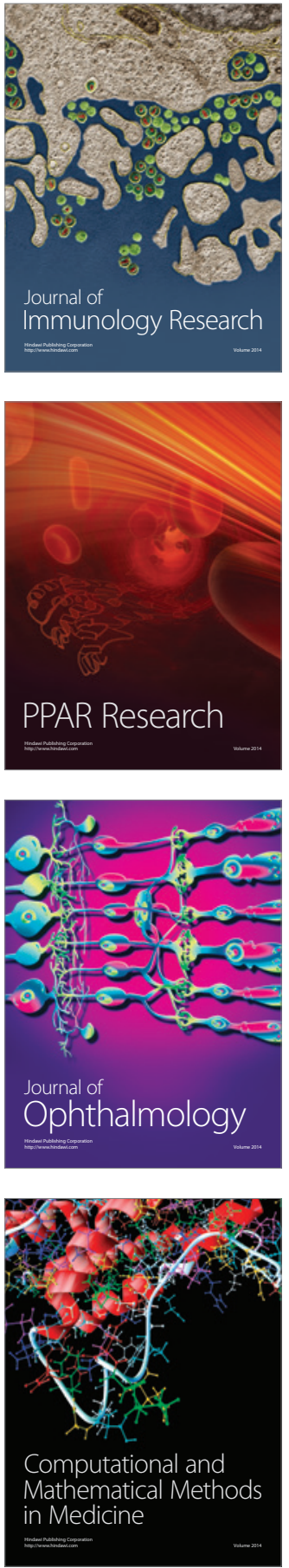

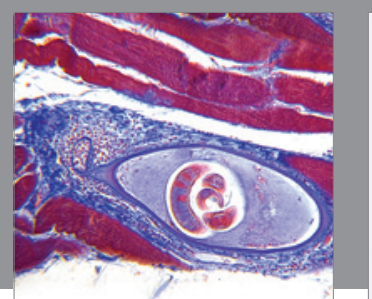

Gastroenterology Research and Practice

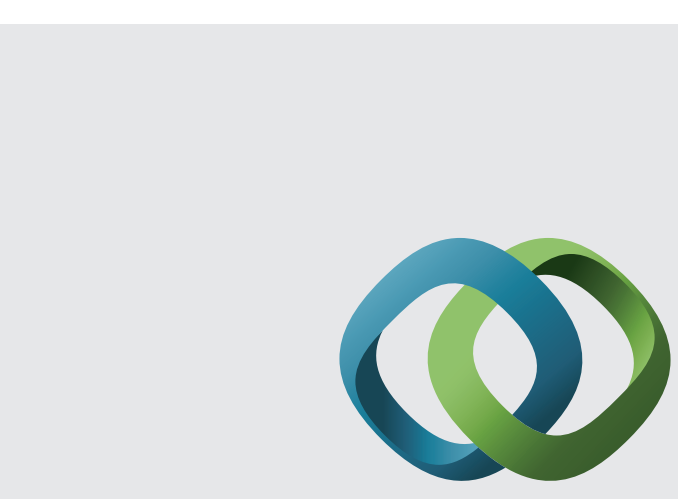

\section{Hindawi}

Submit your manuscripts at

http://www.hindawi.com
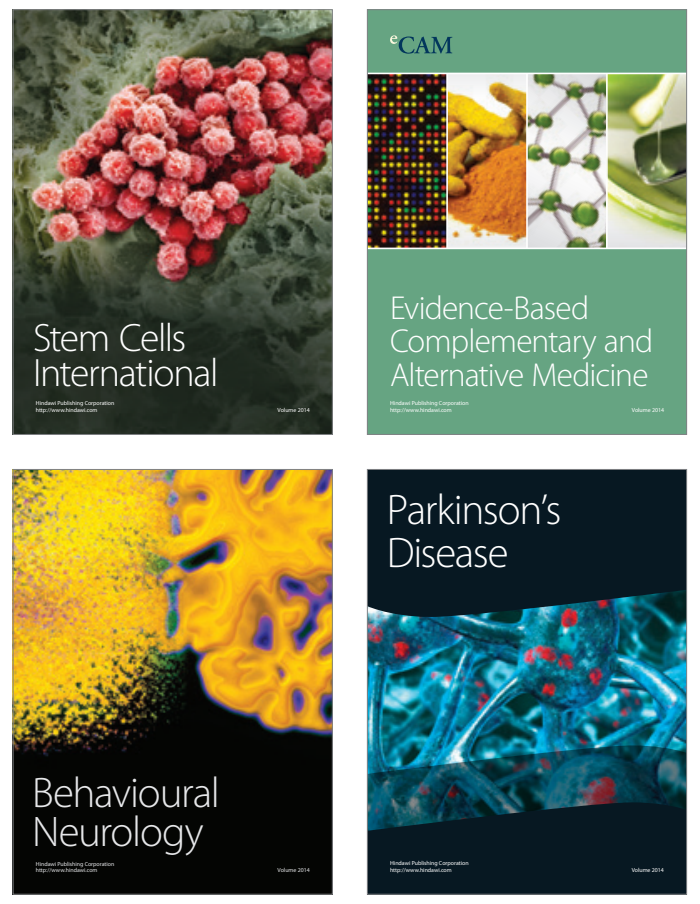
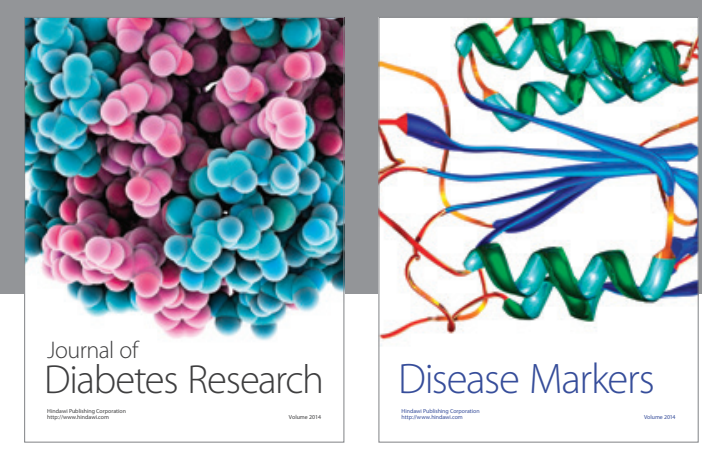

Disease Markers
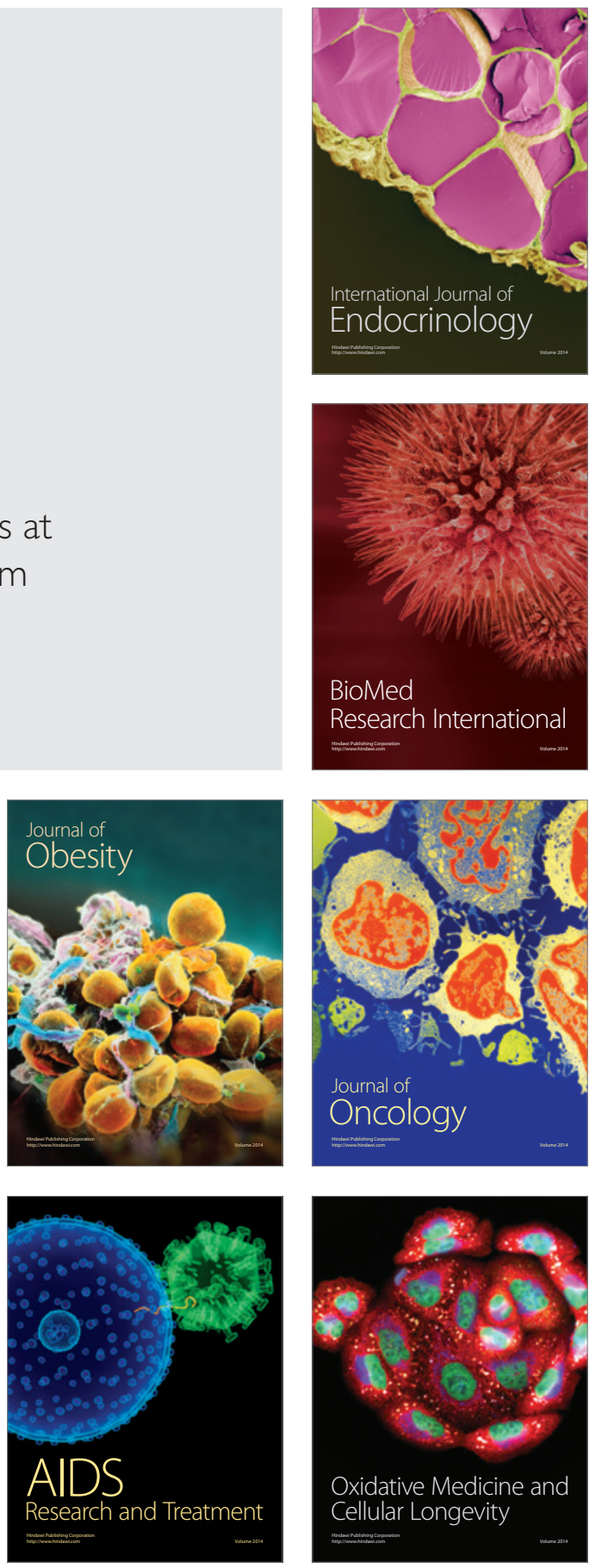